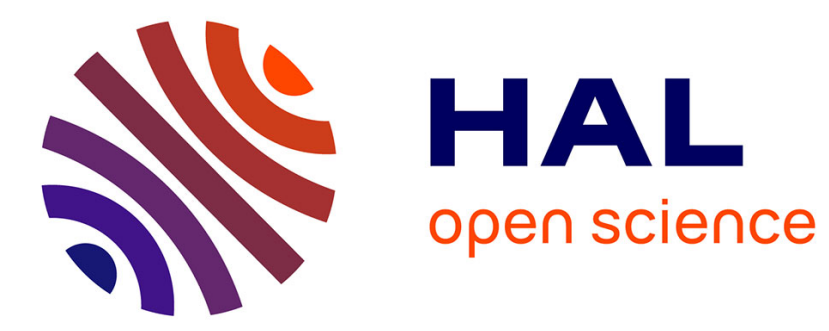

\title{
Etude numérique des convertisseurs héliothermiques tubulaires. Application aux capteurs THEK R. Pasquetti
}

\section{To cite this version:}

R. Pasquetti. Etude numérique des convertisseurs héliothermiques tubulaires. Application aux capteurs THEK. Revue de Physique Appliquée, 1986, 21 (12), pp.785-794. 10.1051/rphysap:019860021012078500 . jpa-00245499

\section{HAL Id: jpa-00245499 https://hal.science/jpa-00245499}

Submitted on 1 Jan 1986

HAL is a multi-disciplinary open access archive for the deposit and dissemination of scientific research documents, whether they are published or not. The documents may come from teaching and research institutions in France or abroad, or from public or private research centers.
L'archive ouverte pluridisciplinaire HAL, est destinée au dépôt et à la diffusion de documents scientifiques de niveau recherche, publiés ou non, émanant des établissements d'enseignement et de recherche français ou étrangers, des laboratoires publics ou privés. 
Classification

Physics Abstracts

$02.60-44.00-86.10 \mathrm{~K}$

\title{
Etude numérique des convertisseurs héliothermiques tubulaires. Application aux capteurs THEK
}

\author{
R. Pasquetti
}

U.A. 1168, Systèmes Energétiques et Transferts Thermiques, Département d'Héliophysique, Université de Provence, Centre St Jérôme, 13397 Marseille Cedex 13, France

(Reçu le 30 septembre 1985, révisé le 3 décembre 1985 et le 10 juillet 1986, accepté le 25 août 1986)

\begin{abstract}
Résumé. - L'étude exposée permet l'évaluation du champ des températures dans les absorbeurs placés au foyer de concentrateurs solaires de type THEK. Ces absorbeurs, tubulaires, plans ou en forme de cavité, sont soumis à un champ d'éclairement non uniforme évoluant dans le temps. Le problème instationnaire de transferts thermiques couplés conduction-rayonnement a été résolu numériquement. On a tenu compte, localement (effet de tube) et globalement (effet de cavité), des phénomènes de réflexions multiples tant pour le rayonnement solaire incident que pour le rayonnement infra-rouge émis par les parois et l'environnement. L'équation de diffusion est intégrée tridimensionnellement suivant un schéma implicite aux différences finies. On présente en dernier lieu un exemple d'application du modèle à un capteur développé dans le cadre du projet THEK 2.
\end{abstract}

\begin{abstract}
We present a study allowing a temperature distribution estimate in the absorbers placed in the focal zone of THEK type reflectors. The front side of these tubular absorbers, of flat or cavity shape, is exposed to a non uniform incident flux distribution, varying with time. The dynamic heat transfer problem, conduction plus radiation, was numerically solved. We took into account the multiple reflection phenomena, locally (pipe action) and totally (cavity action), for solar incident rays and infra-red radiation emited by the cavity and the surroundings. The diffusion equation is integrated using an implicit finite difference method. Lastly, we give an example of application to a solar collector realized in the frame of the THEK 2 project.
\end{abstract}

\section{Introduction.}

L'étude présentée relève des travaux de modélisation de systèmes thermiques complexes où interviennent notamment des phénomènes de transferts couplés conduction-rayonnement ; elle s'inscrit, du point de vue de son domaine d'application, dans l'ensemble des études relatives aux chaudières solaires placées au foyer de concentrateurs (par exemple [1-4]).

1.1 LE CONTEXTE. - Dans le cadre du programme THEK (Thermo Hélio Energie Kilowatt), confié à notre laboratoire par le CNRS (PIRSEM) et l'AFME, ont été réalisés des capteurs à concentration quasi ponctuelle de l'éclairement solaire [5]. Les concentrateurs, de surface de captage $50 \mathrm{~m}^{2}$ et de concentration géométrique de l'ordre de 200 , sont des paraboloïdes approximés par des facettes triangulaires planes. Les convertisseurs ont quelques dizaines de centimètres de diamètre et sont constitués de l'enroulement d'un ou plusieurs tubes formant un disque ou une cavité à symétrie axiale. A " l'avant ", les tubes, peints en noir, présentent un facteur d'absorption élevé pour le rayonnement solaire ; à " l'arrière », un isolant classique (laine de verre) limite les pertes par conduction.

Les convertisseurs sont ainsi soumis à un champ d'éclairement variable dans le temps et l'espace. Un programme de calcul [6] permet, connaissant les géométries du concentrateur et du convertisseur, la détermination de la concentration ponctuelle le long des tubes constituant la chaudière. Figure 1, les variations de cette concentration sont présentées pour la chaudière en forme de cavité développée par la société SEP dans le cadre du projet THEK 2 [7]. La distribution des concentrations étant définie, des études ayant pour objectif,

- l'optimisation du convertisseur [8],

- le calcul, validé expérimentalement, des variations de la température fluide en sortie lors de variations des diverses grandeurs d'entrée [9], ont déjà été conduites dans notre laboratoire.

L'étude proposée ici est relative à une modélisation tridimensionnelle et instationnaire des chaudières solaires de type spirale multitubulaire plane ou en cavité, placées au foyer des concentrateurs. Pour mener à bien cette étude, qui vise à une évaluation 


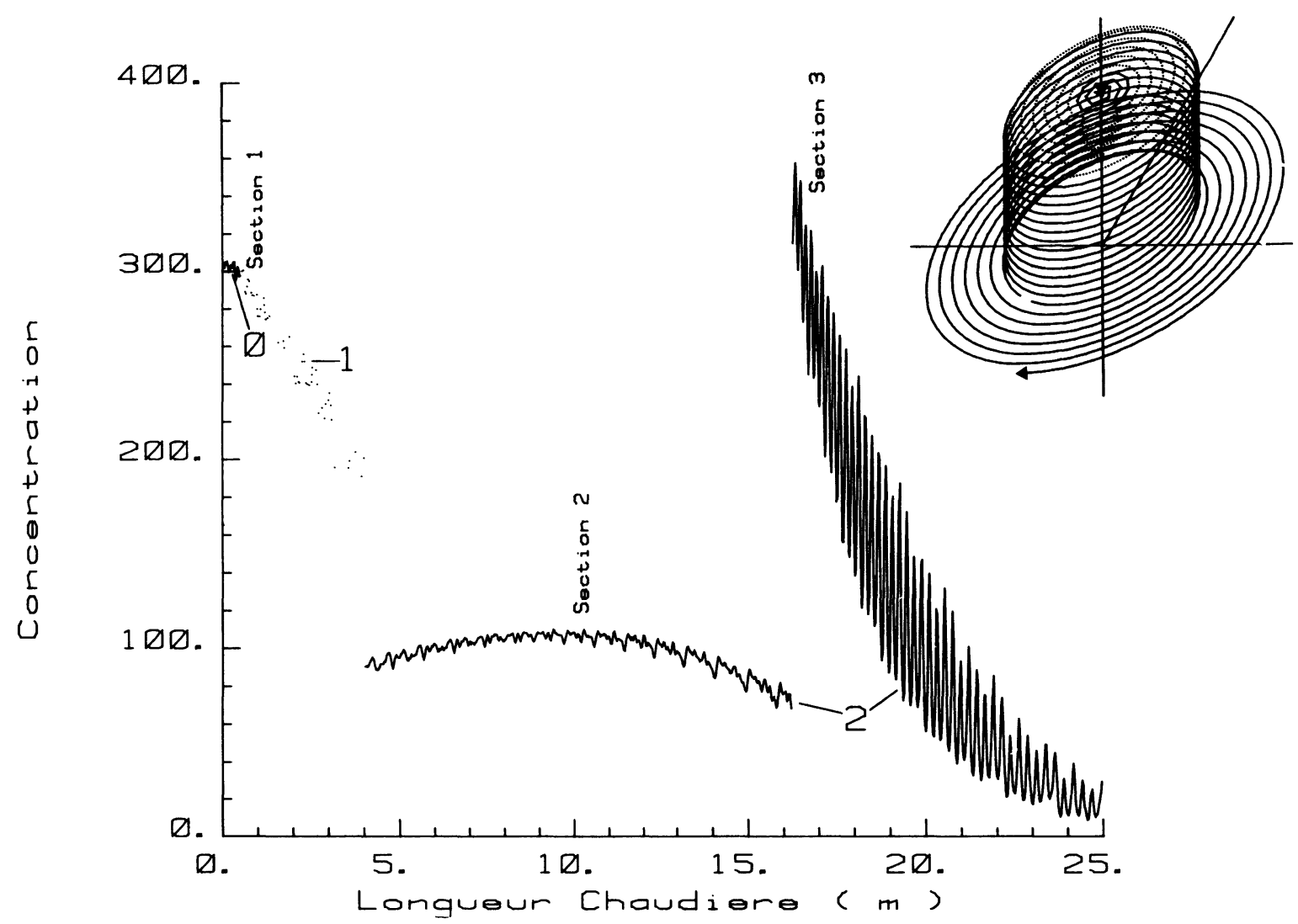

Fig. 1. - Distribution des concentrations le long du tube chaudière ; 0 : boîtier central, 1-2 : tubes d'épaisseur $1 \mathrm{~mm}$ et de diamètres extérieurs 16 et $18 \mathrm{~mm}$ - schéma de la chaudière.

[Local concentration distribution along the absorber pipe ; 0 : central box ; 1-2:1 mm thick and 16-18 mm external diameter pipes ; absorber design.]

des variations du champ des températures, nous avons émis diverses hypothèses simplificatrices. Cidessous, dans la description de la démarche que nous avons suivie, ces hypothèses sont précisées.

1.2 LA DÉMARCHE ET LES HYPOTHÈSES. - La solution a été recherchée en découplant le problème de transfert radiatif localisé, au niveau des « macrorugosités » que constituent les tubes jointifs, du problème de transfert radiatif dans la cavité. Les " résultats locaux » obtenus en premier lieu permettront ainsi de considérer, pour le calcul des échanges dans la cavité, les « surfaces apparentes » de ces tubes, d'où, une résolution relativement classique. Connaissant les éclairements dans la cavité ces mêmes « résultats locaux » permettront la détermination de la condition aux limites, en périphérie de tube, du problème de diffusion. Les équations d'évolution régissant le champ tridimensionnel des températures dans le métal et la variation longitudinale de la température fluide seront intégrées numériquement.

Plus précisément :

Paragraphe 2, considérant des tubes jointifs de même diamètre et infiniment longs, sous les hypothèses,

- d'isothermie,
- de surface grise et diffuse isotrope en réflexion et émission, on détermine, après discrétisation angulaire d'une section de tube, des coefficients opto-géométriques qui permettent le calcul des «flux surfaciques nets » (émis moins absorbé provenant du tube voisin), comme si le tube était isolé dans un environnement à $0 \mathrm{~K}$. Considérant ensuite un éclairement isotrope et uniforme sur la surface «macro-rugueuse", on montre que ces mêmes coefficients opto-géométriques permettent le calcul des flux surfaciques absorbé en périphérie de tube, comme si ce tube était isolé.

Paragraphe 3 , on s'intéresse au calcul des transferts radiatifs dans la cavité de la chaudière. Classiquement :

- on découple problèmes local et global, en considérant comme surfaces d'échange radiatif les surfaces « apparentes » d'éléments tubulaires ;

- on suppose le recouvrement des domaines spectraux correspondant aux rayonnements solaire et I-R émis dans la cavité suffisamment faible pour pouvoir considérer des propriétés optiques du revêtement différentes dans chacun de ces domaines ;

- on néglige, les distances étant faibles, l'influence de la traversée de l'air dans la cavité ; — on émet l'hypothèse, vérifiée en [10], de quasi- 
isotropie à l'émission et à la réflexion de la surface macro-rugueuse.

Le calcul de redistribution des flux solaires est alors classique [11]. Pour celui correspondant au domaine I-R la difficulté réside dans la détermination du flux surfacique « émis » par la surface apparente, fonction des " flux surfaciques nets » en périphérie avant le tube. L'utilisation des coefficients opto-géométriques calculés paragraphe 2 induit en effet une approximation car, ayant été calculés sous l'hypothèse d'isothermie, ils ne prennent en compte que les seuls, mais très influents, effets géométriques.

Paragraphe 4 sont d'abord définies les conditions aux limites du problème de transfert thermique par conduction à l'intérieur des tubes. A cet effet :

- On suppose isotrope le rayonnement solaire ou I-R incident sur les surfaces apparentes, après les réflexions multiples dans la cavité ; les résultats obtenus paragraphe 2 permettent alors la détermination, tenant compte des réflexions multiples locales, de la distribution des éclairements en périphérie de tube.

- Les flux surfaciques de pertes par rayonnement sont évalués, hors de l'hypothèse d'isothermie, par utilisation des coefficients opto-géométriques I-R définis paragraphe 2. Cette évaluation est donc d'autant plus approximative que l'anisothermie devient forte ; cependant les flux solaires interceptés dans ce cas sont élevés relativement aux flux I-R, ce qui rend négligeable l'erreur induite par cette approximation.

- Les flux surfaciques de pertes par convection forcée due au vent, ainsi que ceux relatifs aux pertes par conduction dans l'isolant, sont supposés proportionnels à l'écart entre la température au point considéré et la température ambiante.

- A l'interface métal-fluide, on suppose les flux surfaciques. proportionnels à l'écart entre la température au point considéré et la température fluide ; le régime de l'écoulement est turbulent.

En dernier lieu est formulée l'équation de transport ; concernant le fluide caloporteur, on émet les hypothèses :

— d'incompressibilité, res,

- d'unidimensionnalité du champ des températuble,

- de dissipation d'énergie par viscosité négligea-

- de conduction longitudinale négligeable,

- d'opacité au rayonnement I-R.

Paragraphe 5 est évoquée la procédure numérique d'intégration des équations d'évolution. Connaissant les variations du débit masse, de l'éclairement solaire, des températures ambiante et d'entrée en chaudière, de la vitesse du vent, on détermine les variations du champ total des températures. A chaque pas de temps, après le calcul du champ des éclairements solaires, le schéma d'intégration utilisé (Crank-Nicholson [12]) est inséré dans un processus itératif enchaînant les calculs,
- des flux surfaciques « émis» par les surfaces apparentes connaissant le champ des températures superficielles,

- des éclairements I-R sur ces surfaces,

- des éclairements I-R en périphérie des tubes,

- du champ total des températures,

jusqu'à stabilisation de ce dernier résultat. Un exemple d'application est présenté en dernier lieu, dans le but de montrer les possibilités du modèle mis au point.

\section{Les phénomènes de macro-rugosité tubulaire.}

Les chaudières étudiées sont constituées de tubes jointifs ; il en résulte, entre ces tubes, des " phénomènes locaux » de réflexions multiples, tant pour le rayonnement solaire qu'infra-rouge.

2.1 LE RAYONNEMENT I-R. - Soit 2 tubes jointifs infiniment longs et à température uniforme, dans un environnement à $0 \mathrm{~K}, \theta_{1}$ et $\theta_{2}$ les angles définissant (Fig. 2) 2 génératrices en regard et $\varphi_{\theta}$ la fonction de

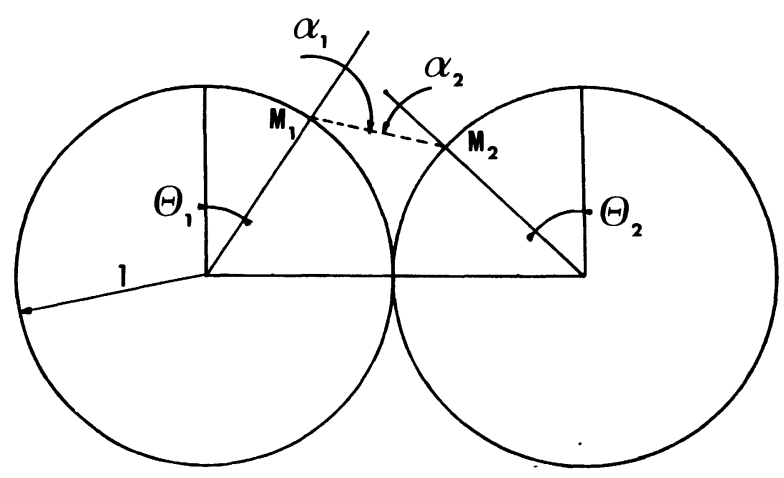

Fig. 2. - Définition géométrique des génératrices en regard $M 1$ et $M 2$.

[Geometrical definition of the opposit generants $M 1$ and M2.]

variation de la radiosité sur les tubes considérés. Soit $\mathrm{d} s_{1}$ et $\mathrm{d} s_{2}$ les surfaces élémentaires correspondant à ces génératrices et $\mathrm{d} s_{1,2}$ la surface d'échange radiatif entre ces surfaces élémentaires. En supposant les tubes gris et isotropes, en émission et réflexion, avec $\varphi_{\mathrm{b}}$ l'émittance du corps noir, $\varepsilon$ et $\rho$ les facteurs d'émission et de réflexion, on a, pour le calcul de la radiosité, l'équation implicite :

$$
\mathrm{d} s_{1} \varphi_{\theta_{1}}=\mathrm{d} s_{1} \varepsilon \varphi_{\mathrm{b}}+\rho \int_{0}^{\pi / 2} \varphi_{\theta_{2}} \mathrm{~d} s_{2,1}
$$

soit

$$
\frac{1}{\rho} \frac{\varphi_{\theta_{1}}}{\varphi_{\mathrm{b}}}-\int_{0}^{\pi / 2} \frac{\varphi_{\theta_{2}}}{\varphi_{\mathrm{b}}} \mathrm{d} f_{1,2}=\frac{\varepsilon}{\rho},
$$

où $\mathrm{d} f_{1,2}$ est le facteur de forme de $\mathrm{d} s_{1}$ à $\mathrm{d} s_{2}$. 
On dispose ainsi d'une équation intégrale en $\varphi_{\theta}$. Par discrétisation du demi-tube en $I$ angles $\Delta \theta$, et en exprimant, suivant la méthode des rectangles, l'intégrale apparaissant en (2), on est conduit à l'expression sous forme discrète :

$$
\frac{1}{\rho} \frac{\varphi_{i}}{\varphi_{\mathrm{b}}}-\sum_{j=1}^{I / 2} \frac{\varphi_{j}}{\varphi_{\mathrm{b}}} f_{i, j}=\frac{\varepsilon}{\rho},
$$

où $f_{i, j}$ est le facteur de forme entre les surfaces $s_{i}$ et $s_{j}$, définies par les angles $\Delta \theta, \theta_{i}=(i-1 / 2) \Delta \theta$ et $\boldsymbol{\theta}_{j}=(j-1 / 2) \Delta \boldsymbol{\theta}$.

On peut exprimer (3) sous la forme d'un système d'équations linéaires :

$$
A \mathbf{Y}=\mathbf{C},
$$

tel que, en notant en minuscules les termes de la matrice $A$ et des vecteurs $\mathbf{C}$ et $\mathbf{Y}$ :

$$
\begin{aligned}
a_{i i} & =\frac{1}{\rho}-f_{i, i} \\
a_{i j} & =-f_{i, j} \\
c_{i} & =\varepsilon / \rho \\
y_{i} & =\varphi_{i} / \varphi_{\mathrm{b}} .
\end{aligned}
$$

La propriété de symétrie du problème étudié induit $f_{i, j}=f_{j, i}$. Sous forme intégrale, les facteurs de forme $f_{i, j}$ intervenant dans la matrice symétrique $A$ peuvent s'exprimer, par unité de longueur, suivant :

$$
f_{i, j}=\frac{1}{\Delta \theta} \int_{\theta_{i}-\Delta \theta / 2}^{\theta_{i}+\Delta \theta / 2} \int_{\theta_{j}-\Delta \theta / 2}^{\theta_{j}+\Delta \theta / 2} f\left(\theta_{1}, \theta_{2}\right) \mathrm{d} \theta_{1} \mathrm{~d} \theta_{2},
$$

avec

$$
f\left(\theta_{1}, \theta_{2}\right)=\frac{1}{2 M_{1} M_{2}} \max \left[\cos \alpha_{1}\left(\theta_{1}, \theta_{2}\right), 0\right] \max \left[\cos \alpha_{2}\left(\theta_{1}, \theta_{2}\right), 0\right]
$$

En notant $p_{i}$ le flux surfacique net, c'est-à-dire la différence algébrique entre flux surfaciques émis et absorbés en $i$, on a :

$$
p_{i}=\varepsilon \varphi_{\mathrm{b}}-\left(\varphi_{i}-\varepsilon \varphi_{\mathrm{b}}\right) \frac{\varepsilon}{\rho},
$$

soit encore $(\operatorname{car} \varepsilon=1-\rho)$

$$
\frac{p_{i}}{\varphi_{\mathrm{b}}}=\frac{\varepsilon}{\rho}\left(1-\frac{\varphi_{i}}{\varphi_{\mathrm{b}}}\right)
$$

Avec $\omega_{i}=\frac{p_{i}}{\varphi_{\mathrm{b}}}$ on a, sous forme matricielle $(\Omega$ vecteur de composantes $\omega_{i}$ et 1 le vecteur de composantes unitaires) :

$$
\Omega=\frac{\varepsilon}{\rho}(\mathbf{1}-\mathbf{Y})
$$

D'après (4) on peut écrire

$$
A \Omega=\frac{\varepsilon}{\rho}[A \cdot \mathbf{1}-\mathbf{C}],
$$

soit

$$
A \Omega=\mathbf{B},
$$

avec

$$
b_{i}=\frac{\varepsilon}{\rho}\left(1-\sum_{j=1}^{I / 2} f_{i, j}\right)
$$

Les coefficients $\omega_{i}$, bornés entre 0 et $\varepsilon$, sont des coefficients opto-géométriques. Ils permettent, substitués au facteur d'émission, le calcul des flux surfaciques « perdus » par le tube, à l'élément de surface $i$, comme si ce tube était isolé dans un environnement à $0 \mathrm{~K}$.

2.2 LE RAYONNEMENT SOLAIRE OU INFRA-ROUGE INCIDENT. - Soit maintenant $\varphi_{\theta 1}$ et $\varphi_{\theta 2}$ les éclairements sur éléments de surface $\mathrm{d} s_{1}$ et $\mathrm{d} s_{2}$ repérés par les angles $\theta_{1}$ et $\theta_{2}$ et $\varphi_{\mathrm{e}}$ l'éclairement, avant les phénomènes locaux de réflexions multiples, supposé uniforme et isotrope. On a alors :

$$
\mathrm{d} s_{1} \varphi_{\theta_{1}}=\int_{0}^{\pi / 2} \rho \varphi_{\theta_{2}} \mathrm{~d} s_{2,1}+\left(\mathrm{d} s_{1}-\int_{0}^{\pi / 2} \mathrm{~d} s_{1,2}\right) \varphi_{\mathrm{e}}
$$


soit

$$
\frac{1}{\rho} \frac{\varphi_{\theta_{1}}}{\varphi_{\mathrm{e}}}-\int_{0}^{\pi / 2} \frac{\varphi_{\theta_{2}}}{\varphi_{\mathrm{e}}} \mathrm{d} f_{1,2}=\frac{1}{\rho}\left(1-\int_{0}^{\pi / 2} \mathrm{~d} f_{1,2}\right),
$$

et sous forme discrétisée

$$
\frac{1}{\rho} \frac{\varphi_{i}}{\varphi_{\mathrm{e}}}-\sum_{j=1}^{I / 2} \frac{\varphi_{j}}{\varphi_{\mathrm{e}}} f_{i, j}=\frac{1}{\rho}\left(1-\sum_{j=1}^{I / 2} f_{i, j}\right) .
$$

En multipliant les deux membres de cette égalité par $\varepsilon$, on fait apparaître des coefficients opto-géométriques qui permettent un calcul direct du flux surfacique absorbé par l'élément de surface $i$. Ces coefficients sont solution de :

$$
A \Omega=\mathbf{B},
$$

avec

$$
\omega_{i}=\varepsilon \frac{\varphi_{i}}{\varphi_{\mathrm{e}}}
$$

et les coefficients de $A$ et $\mathbf{B}$ définis en 2.1.

Ainsi, suivant nos hypothèses, la résolution du système (15) permet la détermination des variations d'une grandeur s'apparentant à un facteur d'émission ou d'absorption. Connaissant l'éclairement $\varphi_{\mathrm{e}}$ il est alors possible de calculer simplement la densité de flux absorbé en $i$. On peut de plus définir un facteur de réflexion $\left(\rho_{\mathrm{a}}\right)$ ou d'absorption $\left(\varepsilon_{\mathrm{a}}\right)$ apparent d'après :

$$
\begin{gathered}
\varepsilon_{\mathrm{a}} \varphi_{\mathrm{e}}=\sum_{j=1}^{I / 2} \omega_{j} \Delta \theta \varphi_{\mathrm{e}}, \\
\varepsilon_{\mathrm{a}}=\frac{\pi}{I} \sum_{j=1}^{I / 2} \omega_{j} \text { et } \rho_{\mathrm{a}}=1-\varepsilon_{\mathrm{a}} .
\end{gathered}
$$

Figure 3 sont présentées les variations du coefficient $\omega$ en fonction de l'angle $\theta$ pour différents facteurs de réflexion. En regard de chaque courbe apparaissent les valeurs de $\varepsilon$ et $\varepsilon_{\mathrm{a}}$. Le facteur de réflexion $\rho$ (ou d'absorption $\varepsilon$ ) intervenant comme paramètre, on distinguera ci-dessous 2 domaines spectraux correspondant au rayonnement solaire et I-R. On distinguera notamment les coefficients $\omega, \rho_{\mathrm{a}}$ pour le rayonnement I-R des coefficients $\omega^{\prime}, \rho_{\mathrm{a}}^{\prime}$ pour le rayonnement solaire.

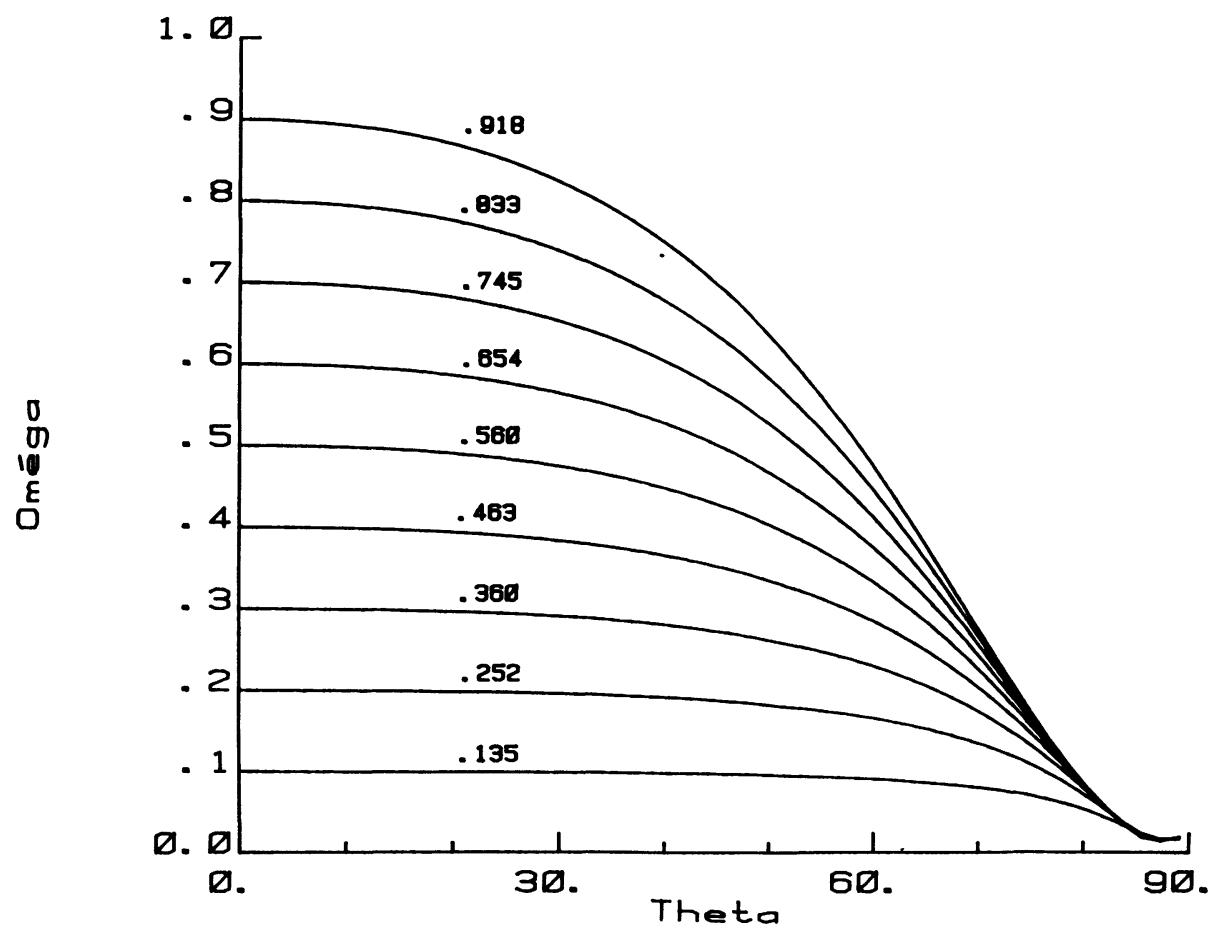

Fig. 3. - Variations avec l'angle $\theta$ et pour différents facteurs de réflexion du coefficient $\omega$.

[Coefficient $\omega$ versus $\theta$ angle for different reflectivities.] 


\section{Les couplages radiatifs dans la cavité.}

Pour traiter ce problème des couplages radiatifs dans la cavité de la chaudière on va d'une part procéder à un découpage longitudinal des tubes qui la constituent et d'autre part leur substituer la surface réglée par les diamètres pour lesquels l'angle $\theta$ est égal à $\pi / 2$ (Fig. 2). On supposera la surface « macrorugueuse " parfaitement isotrope, en émission et réflexion, et " indépendamment grise " pour chacun des domaines spectraux considérés.

3.1 LE RAYONNEMENT I-R. - En premier lieu il faut, d'après les résultats obtenus paragraphe 2, exprimer la densité de flux $P_{\mathrm{m}}$ " émis " par l'élément de tube $\mathrm{m}$. Sous forme discrétisée et en abandonnant l'hypothèse d'isothermie :

$$
P_{\mathrm{m}}=\frac{\pi}{I} \sum_{j=1}^{I / 2} \omega_{j} \varphi_{\mathrm{b}_{j}}
$$

Soit $S_{\mathrm{m}}$ la surface correspondant au tube m, $S_{\mathrm{m}, \mathrm{n}}$ la surface d'échange radiatif entre $S_{\mathrm{m}}$ et $S_{n}, S_{\mathrm{m}, \mathrm{o}}$ la surface d'échange radiatif entre $S_{\mathrm{m}}$ et l'ouverture de la cavité, $\Phi_{\mathrm{m}}$ la radiosité en $S_{\mathrm{m}}, \Phi_{\mathrm{o}}$ la densité de flux à l'ouverture (assimilée au corps noir à la température ambiante) ; classiquement on écrit, sous forme discrétisée :

$$
S_{\mathrm{m}} \Phi_{\mathrm{m}}=S_{\mathrm{m}} P_{\mathrm{m}}+\rho_{\mathrm{a}}\left[S_{\mathrm{m}, \mathrm{o}} \Phi_{\mathrm{o}}+\sum_{n=1}^{M} S_{n, \mathrm{~m}} \Phi_{\mathrm{n}}\right] \text {. }
$$

Sous forme matricielle :

$$
S \Phi=\mathbf{E},
$$

avec, en notant en minuscules les éléments de la matrice $S$ et du vecteur $\mathbf{E}$ :

$$
\begin{aligned}
& s_{\mathrm{mm}}=S_{\mathrm{m}} / \rho_{\mathrm{a}}-S_{\mathrm{m}, \mathrm{m}} \\
& s_{\mathrm{m} n}=-S_{\mathrm{m}, n} \\
& e_{\mathrm{m}}=S_{\mathrm{m}} P_{\mathrm{m}} / \rho_{\mathrm{a}}+S_{\mathrm{m}, \mathrm{o}} \Phi_{\mathrm{o}} .
\end{aligned}
$$

La résolution de (20) a pour résultat l'ensemble des radiosités dans la cavité : on en déduit les densités $R_{\mathrm{m}}$ de flux I-R incident sur les surfaces $S_{\mathrm{m}}\left(\mathbf{R}, \Phi, \mathbf{P}\right.$, vecteurs de composantes $\left.R_{\mathrm{m}}, \Phi_{\mathrm{m}}, P_{\mathrm{m}}\right)$ :

$$
\mathbf{R}=(\boldsymbol{\Phi}-\mathbf{P}) / \rho_{\mathrm{a}} .
$$

Le calcul des surfaces d'échange $S_{\mathrm{m}, n}$ doit être effectué numériquement ; les surfaces $S_{\mathrm{m}}$ et $S_{n}$ sont des éléments d'une même ou de deux spirales que l'on caractérise, dans le programme mis au point, par les coordonnées cylindriques (non affectées d'un modulo $2 \pi$ ) de leurs points extrêmes. Il s'agit en toute rigueur d'une intégrale quadruple; nous l'avons approximée par une intégrale double en nous limitant à une seule discrétisation longitudinale des surfaces « apparentes». Le résultat peut toutefois être faussé aux « angles » de la cavité ; pour éviter cet inconvénient nous profitons du fait qu'en calculant directement les surfaces $S_{\mathrm{m}, \mathrm{o}}$ on peut calculer indirectement ce résultat en déterminant le complément à $S_{\mathrm{m}}$ de la somme des autres surfaces d'échange.

3.2 LE RAYONNEMENT SOLAIRE. - Soit maintenant $\Phi_{\mathrm{m}}^{\prime}$ l'éclairement solaire parvenant directement en $\mathrm{m}$ et $Q_{\mathrm{m}}$ celui résultant des phénomènes de réflexions multiples. Avec $\rho_{\mathrm{a}}^{\prime}$ le facteur de réflexion apparent, on a :

$$
S_{\mathrm{m}} Q_{\mathrm{m}}=S_{\mathrm{m}} \Phi_{\mathrm{m}}^{\prime}+\rho_{\mathrm{a}}^{\prime} \sum_{n=1}^{M} Q_{n} S_{n, \mathrm{~m}},
$$

soit

$$
S^{\prime} \mathbf{Q}=\mathbf{E}^{\prime},
$$

avec pour la matrice $S^{\prime}$ et le vecteur $\mathbf{E}^{\prime}$ :

$$
\begin{aligned}
s_{\mathrm{mm}}^{\prime} & =S_{\mathrm{m}} / \rho_{\mathrm{a}}^{\prime}-S_{\mathrm{m}, \mathrm{m}} \\
s_{\mathrm{m} n}^{\prime} & =-S_{\mathrm{m}, n} \\
e_{\mathrm{m}}^{\prime} & =S_{\mathrm{m}} \Phi_{\mathrm{m}}^{\prime} / \rho_{\mathrm{a}}^{\prime} .
\end{aligned}
$$

Connaissant le champ des éclairements (Fig. 1) et celui des températures superficielles les expressions ci-dessus formulées permettent donc le calcul, pour chaque surface apparente, des éclairements solaire et I-R.

\section{Le transfert de chaleur au fluide caloporteur.}

Ce transfert thermique est régi par une équation de diffusion à l'intérieur des tubes chaudière et par une équation de transport pour le fluide caloporteur.

4.1 LE TRANSFERT CONDUCTIF. - En considérant infini le rayon de courbure des axes des tubes, relativement à leur diamètre, pour traiter le problème de diffusion il suffit d'intégrer numériquement l'équation de la chaleur sur un domaine cylindrique. A cet effet, on complémente les discrétisations angulaire et longitudinale précédemment définies (paragraphes 2 et 3 ) par une discrétisation radiale. Il en résulte une discrétisation tridimensionnelle permettant de mettre en œuvre la méthode numérique exposée en [13] et évoquée, pour cette étude particulière, paragraphe 5 . Cette méthode garantit, spatialement et temporellement, une précision au second ordre. Elle permet de tenir compte des non-linéarités dues aux variations des coefficients thermo-physiques avec la température et, aux limites, à celles dues aux pertes par rayonnement.

4.1.1 Condition aux limites en périphérie avant. - Il s'agit d'une condition de type mixte Neumann-Fourier variable spatialement (longitudinalement et angulairement), temporellement, et dépendante du champ des températures superficielles dans l'ensemble de la cavité (couplage conduction-rayonnement). 
Avec $\mu$ la densité de flux résultant d'un bilan entre :

- pertes thermiques par rayonnement I-R et convection,

- rayonnement solaire absorbé,

- rayonnement I-R absorbé,

avec $\lambda$ la conductivité du métal, $r_{\mathrm{e}}$ le rayon extérieur du tube (rayon courant $r$ ) et $T$ la température dans le métal :

$$
\lambda\left(\frac{\partial T}{\partial r}\right)_{r_{\mathrm{e}}}=\mu
$$

Dans une section déterminée du tube chaudière, avec $R_{\mathrm{m}}$ et $Q_{\mathrm{m}}$ calculés comme en (21) et (23), $h_{\mathrm{cv}}$ le coefficient de pertes par convection et $T_{\mathrm{e}}$ la température de l'environnement, d'après l'ensemble des résultats précédemment obtenus, on a, sous forme discrète :

$\mu_{i}=\omega_{i}^{\prime} Q_{\mathrm{m}}+\omega_{i}\left(R_{\mathrm{m}}-\varphi_{\mathrm{b}_{\mathrm{i}}}\right)-h_{\mathrm{cv}}\left(T_{\mathrm{i}}-T_{\mathrm{e}}\right)$.

4.1.2 Condition aux limites en périphérie arrière. L'isolant a simplement été assimilé à une résistance thermique ; avec $h_{\mathrm{IS}}$ le coefficient de transfert thermique :

$$
\lambda\left(\frac{\partial T}{\partial r}\right)_{r_{\mathrm{e}}}=\frac{-2}{\pi} h_{\mathrm{IS}}\left(T-T_{\mathrm{e}}\right) .
$$

4.1.3 Condition aux limites à l'interface métal-fluide. - Avec $h_{\mathrm{MF}}$ le coefficient de transfert métal-fluide, $T_{\mathrm{f}}$ la température du fluide dans la section de tube considérée et $r_{\mathrm{o}}$ le rayon intérieur :

$$
\lambda\left(\frac{\partial T}{\partial r}\right)_{r_{\mathrm{o}}}=h_{\mathrm{MF}}\left(T-T_{\mathrm{f}}\right) .
$$

Pour le calcul du coefficient $h_{\mathrm{MF}}$ nous avons utilisé la classique formule de Colburn, qui en toute rigueur suppose une température film-fluide uniforme, et tenu compte de la dépendance des grandeurs thermophysiques avec la température.

4.2 L'ÉQUATION DE TRANSPORT. - Avec $\ddot{m}$ le débit massique, $C_{\mathrm{f}}$ la chaleur spécifique du fluide caloporteur, $C_{t}$ la capacité thermique du fluide par unité de longueur et $x$ l'abscisse curviligne suivant le tube :

$$
C_{\mathrm{t}} \frac{\partial T_{\mathrm{f}}}{\partial t}=2 r_{\mathrm{o}} \int_{0}^{\pi} h_{\mathrm{MF}}\left(T\left(r_{\mathrm{o}}, \theta\right)-T_{\mathrm{f}}\right) \mathrm{d} \theta-\dot{m} C_{\mathrm{f}} \frac{\partial T_{\mathrm{f}}}{\partial x} .
$$

Par approximation au second ordre de la dérivée spatiale, avec $T_{\mathrm{em}}$ et $T_{\mathrm{sm}}$ les températures d'entrée et de sortie à l'élément $\mathrm{m}$ :

$$
\frac{C_{\mathrm{t}}}{2}\left(\frac{\partial T_{\mathrm{em}}}{\partial t}+\frac{\partial T_{\mathrm{sm}}}{\partial t}\right)=2 \Delta \theta r_{\mathrm{o}} \sum_{j=1}^{I} h_{\mathrm{MF}}\left(T_{j}-\frac{T_{\mathrm{em}}+T_{\mathrm{sm}}}{2}\right)-\dot{m} C_{\mathrm{f}} \frac{T_{\mathrm{sm}}-T_{\mathrm{em}}}{\Delta x} .
$$

Comme en 4.1 on a approximé la dérivée temporelle par différences finies centrées au second ordre.

\section{La procédure numérique. Un exemple d'applica-} tion.

5.1 LA PROCÉDURE NUMÉRIQUE. - Un organigramme du programme mis au point est présenté figure 4. On distingue essentiellement deux phases ; on effectue d'abord les calculs d'initialisation puis on intègre numériquement les équations d'évolution.

Dans la phase d'initialisation sont notamment déterminés :

- les coefficients $\omega_{i}$ et $\omega_{i}^{\prime}$,

- des paramètres géométriques simples caractéristiques de la chaudière et de la discrétisation qui en a été faite,

- les surfaces d'échange radiatif,

- les concentrations ponctuelles moyennes, avant puis après prise en compte des réflexions multiples, par résolution de (23),
- une forme préconditionnée de la matrice $S$ pour le calcul des flux I-R incidents par résolution de (20) suivant la méthode de Cholesky.

Dans la seconde phase, pour intégrer les équations d'évolution on utilise un schéma implicite de CrankNicholson avec itération sur l'algorithme pour garantir, compte tenu des non-linéarités, la précision au second ordre. Comme figuré sur l'organigramme, au niveau de ce calcul, le problème est en fait bidimensionnel et les radiosités, ainsi que les flux longitudinaux, sont supposés connus.

Le problème tridimensionnel est donc résolu, à chaque instant, par une méthode consistant à itérer sur les résolutions de problèmes bidimensionnels jusqu'à stabilisation de l'ensemble des températures. Cette stabilisation suppose celle des flux conductifs et radiatifs et le champ de températures résultant est donc solution du problème global de transfert thermique couplé. Une telle démarche, dictée par la nature du problème traité - cylindrique à gradients thermiques importants dans la section et faibles 


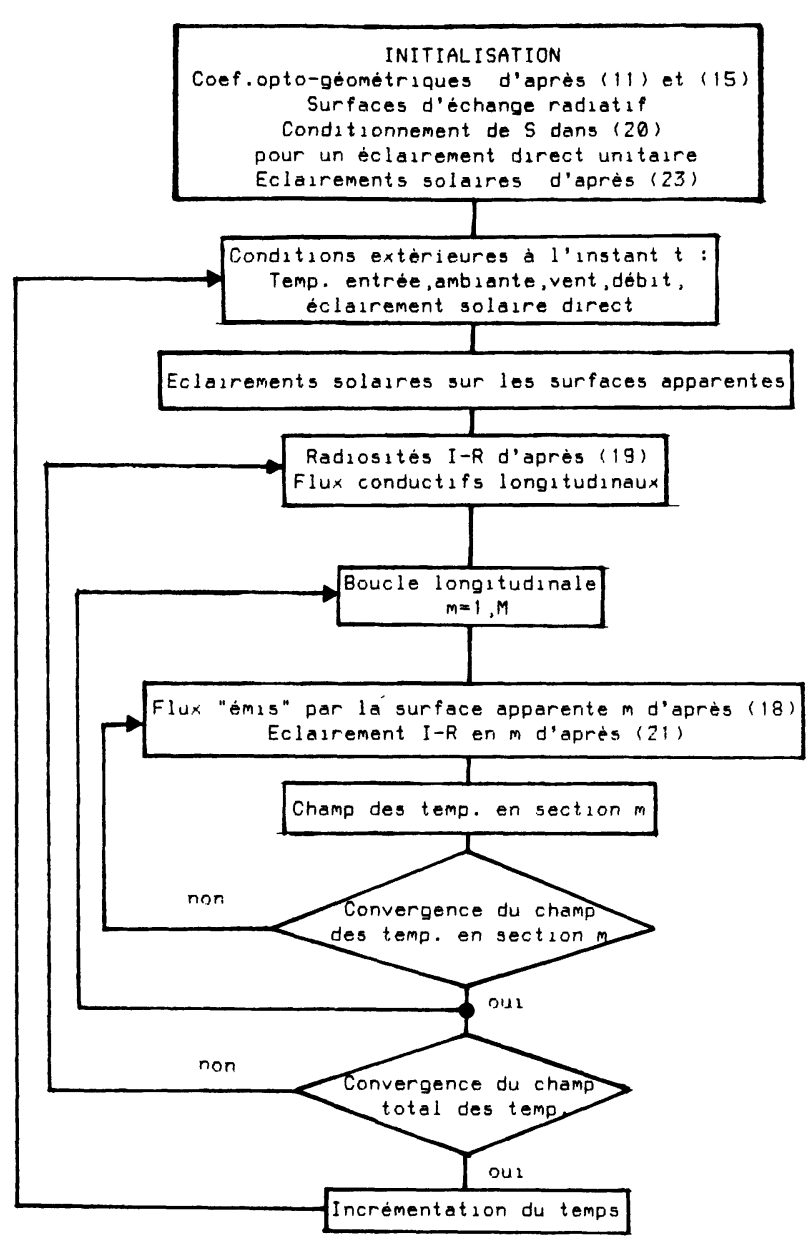

Fig. 4. - Organigramme.

[Flow chart.]

longitudinalement - autorise un gain appréciable en vitesse d'exécution et en encombrement mémoire, les matrices manipulées étant de plus faible dimension et creuses.

5.2 UN EXEMPLE D'APPLICATION. - Figure 5 est donné un exemple de résultat. On y a représenté les variations de la température fluide et de trois températures dans le métal, en périphérie externe pour $\theta=0$ et $180^{\circ}$ et interne pour $\theta=0$, le long des tubes de la chaudière lors d'un échelon d'éclairement solaire $200-800 \mathrm{~W} / \mathrm{m}^{2}$. La température en entrée de chaudière a été prise égale à $260^{\circ} \mathrm{C}$, la température ambiante à $20^{\circ} \mathrm{C}$, le débit à $800 \mathrm{l} / \mathrm{h}$ et la vitesse du vent à $2 \mathrm{~m} / \mathrm{s}$. Le fluide caloporteur est du GilothermTH.

L'absorbeur est celui d'un des capteurs de la centrale THEK 2. Il s'agit d'une chaudière en cavité comportant un boîtier central et 2 tubes en acier inoxydable d'épaisseur $1 \mathrm{~mm}$. La distribution des concentrations ponctuelles le long de la chaudière est celle représentée figure 1. En chaque section on a considéré 32 températures dans le métal, 4 radialement et 8 angulairement, et 3102 pour l'ensemble de l'absorbeur: le fond de la cavité a été discrétisé longitudinalement en 39 éléments, les parois cylindriques en 48 et la collerette en 7 .
En différentes sections du tube, spécifiées figure 1, on a représenté figure 6 les champs de températures obtenus lors de la simulation; pour faciliter la représentation l'angle $\theta$ est porté en abscisse et l'épaisseur métallique en ordonnée. La mise en évidence de gradients thermiques importants et de températures superficielles élevées est notamment intéressante pour les études de vieillissement des peintures absorbantes, ou du fluide caloporteur, et de tenue mécanique des tubes constitutifs de la chaudière.

\section{Conclusion.}

Nous avons décrit un modèle permettant le calcul du champ des températures dans un convertisseur héliothermique tubulaire en forme de cavité, soumis à un champ de densités de flux variable dans l'espace et le temps. Ce modèle procède par intégration numérique d'une équation non linéaire de diffusion avec pour conditions aux limites les résultats d'un problème de transfert radiatif.

Pour mener à bien cette étude diverses hypothèses ont été émises. Un affinement du modèle pourrait être obtenu en revenant sur celles ayant la plus grande incidence sur les champs de températures calculés. Il serait en particulier souhaitable :

1) d'affiner l'hypothèse d'isotropie des éclairements solaires sur les tubes de la chaudière, car ces éclairements régissent de manière prépondérante le champ des températures, les flux de pertes étant relativement peu importants. Pour les chaudières planes l'hypothèse $\mathrm{d}$ 'isotropie est probablement acceptable mais elle devient plus critiquable pour les chaudières en cavité et à revêtement très absorbant. Dans le cas des chaudières cylindriques le champ des températures en section de tube est certainement sensiblement asymétrique dans les parties non planes du convertisseur, ce que ne prévoit pas le modèle.

Pour améliorer efficacement la formulation présentée il est indispensable de disposer de données complémentaires concernant le rayonnement parvenant directement dans la cavité, après réflexion sur le concentrateur. La connaissance des variations de la concentration le long des tubes chaudière et perpendiculairement $(\theta=0)$ aux surfaces apparentes de ces tubes (Fig. 1) ne donne en effet pas d'information concernant la direction des rayons incidents. Supposons donc connue la dépendance de ces concentrations avec l'angle polaire $\left(-90^{\circ}<\theta<90^{\circ}\right)$; un calcul plus précis devient envisageable pour le rayonnement solaire directement absorbé et l'hypothèse d'isotropie n'a plus à être émise que pour le rayonnement solaire ayant été réfléchi au moins une fois dans la cavité. Cette hypothèse devient alors plus acceptable et moins critique, car l'énergie mise en jeu de par les phénomènes de réflexions multiples est, pour les chaudières à revêtement très absorbant, relativement faible ;

2) de formuler de manière moins sommaire le transfert thermique à l'interface métal-fluide. Différents auteurs ont mené des études théoriques et 

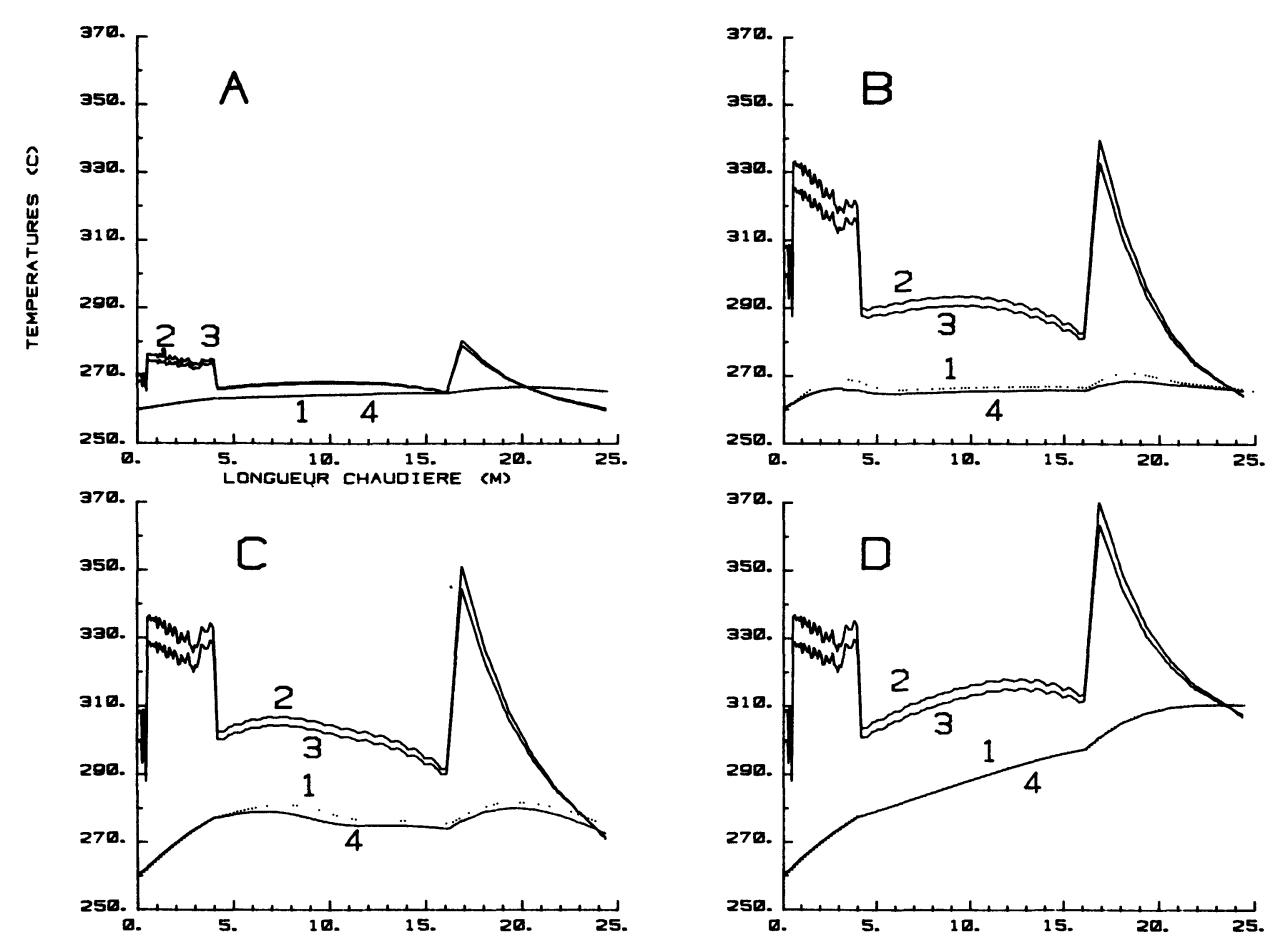

Fig. 5. - Variations le long des tubes chaudière des températures fluide (1), métal avant externe (2) et interne (3), métal arrière externe (4) à $t=-1^{\prime \prime}(\mathrm{A}),+2^{\prime \prime}(\mathrm{B}),+8^{\prime \prime}(\mathrm{C})$ et $+120^{\prime \prime}(\mathrm{D})$ lors d'un échelon d'éclairement 200-800 W/m² à $t=0$.

[Temperature variations along the absorber pipes for fluid (1), metal's front external (2) and internal (3) side, metal's back external (4) side, at $t=-1^{\prime \prime}(\mathrm{A}),+2^{\prime \prime}(\mathrm{B}),+8^{\prime \prime}(\mathrm{C})$ and $+120^{\prime \prime}(\mathrm{D})$ after an incident power step at $t=0$.]

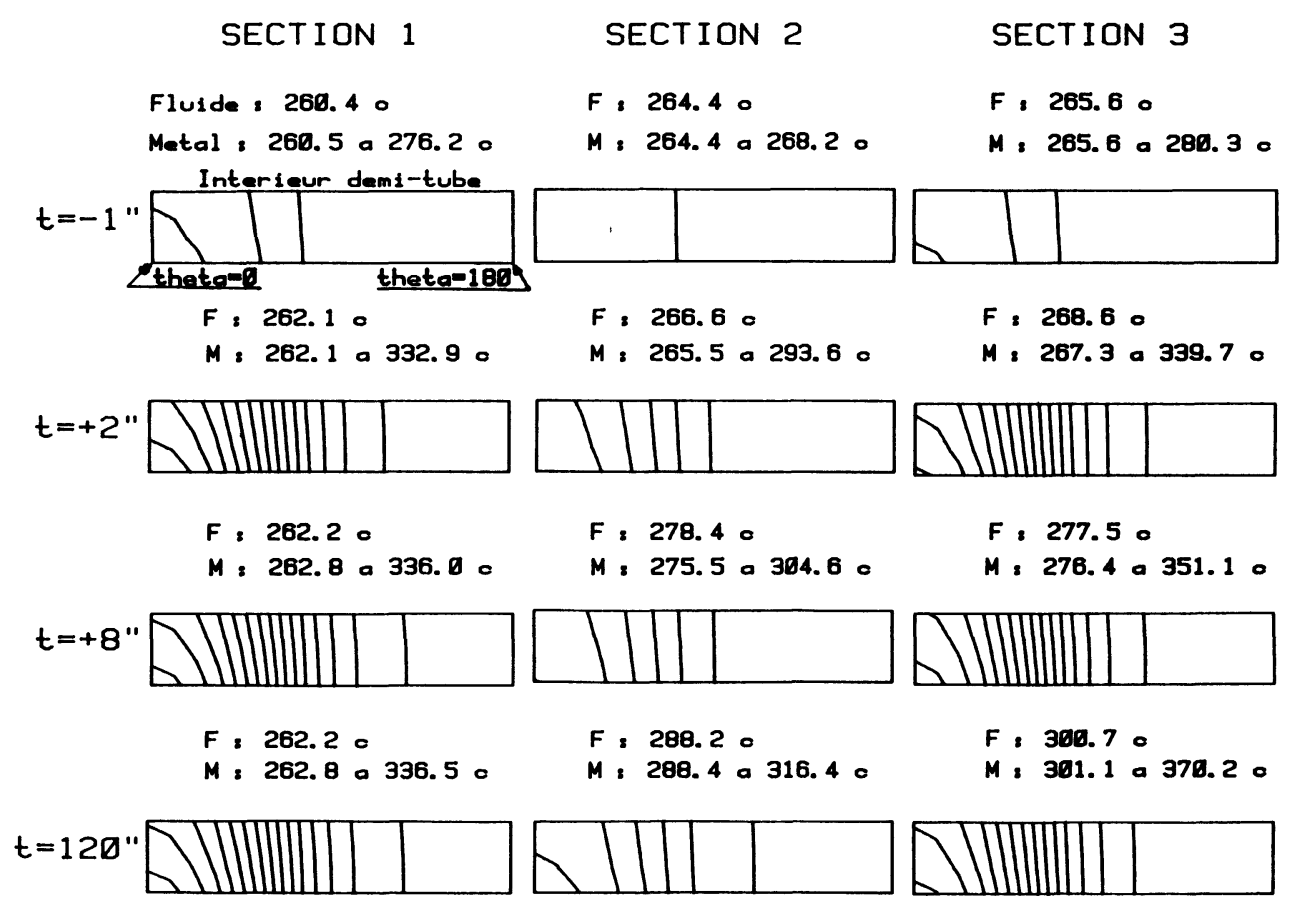

Fig. 6. - Isothermes en différentes sections indiquées figure 1 et à différents instants $t$ lors d'un échelon d'éclairement 200-800 W/ $/ \mathrm{m}^{2}$ à $t=0$. Pour chaque section sont précisées les températures maximale et minimale du métal ainsi que la température du fluide. Les isothermes sont des multiples de $5^{\circ} \mathrm{C}$. Les températures sont décroissantes de $\theta=0^{\circ}$ à $\theta=180^{\circ}$.

[Isotherms for different sections specified in figure 1 and for different delays after an incident power step $200-800 \mathrm{~W} / \mathrm{m}^{2}$ at $t=0$. For every section maximal and minimal metal temperatures and the fluid temperature are specified. Isotherms are multiples of $5^{\circ} \mathrm{C}$. Temperatures are decreasing from $\theta=0^{\circ}$ to $\theta=180^{\circ}$.] 
expérimentales d'écoulements cylindriques soumis à des conditions aux limites variables sur leur circonférence (par exemple [14-17]) ou avec le temps (par exemple [18-19]). Il apparaît notamment que si l'utilisation d'une formule de type Colburn (DittusBoelter), en régime turbulent, peut s'avérer satisfaisante pour calculer l'échauffement du fluide par utilisation d'un nombre de Nusselt moyen, le calcul précis du flux surfacique périphérique met en évidence un nombre de Nusselt local, variable angulairement [20]. Connaissant le champ exact des températures film-fluide le calcul du flux transféré au fluide est donc correct mais le fait de considérer un coefficient de transfert indépendant de l'angle polaire induit, dans notre étude, une approximation dans la connaissance de ce champ.

Une amélioration pourrait toutefois être mise en œuvre, au moins pour le régime stationnaire, car l'itération interne, sur l'algorithme de résolution de l'équation de la chaleur et de transport fluidique dans une section de tube (Fig. 4), peut, sous la réserve d'une "bonne convergence », permettre une résolution numérique dans le fluide couplée à celle dans le métal.

\section{Bibliographie}

[1] Boulanger, F., Evaluation des pertes de la chaudière de la centrale solaire expérimentale THEMIS. EDF. Rapport HP/55/THEM 78-38 (1978).

[2] Wintrebert, G., Blay, D., Ortiz, F., Etude d'un récepteur solaire à haute température pour cycle thermodynamique à gaz. Colloque CNRS : Systèmes solaires thermodynamiques. Marseille (1980).

[3] Demont, P., Huetz-Aubert, M., Tran Nguyen, M., Conception thermique et physique d'un élément capteur haute température haute concentration. Colloque CNRS : Systèmes solaires thermodynamiques. Marseille (1980).

[4] Soufiani, A., Etude des transferts thermiques intervenant dans la chaudière de la centrale solaire THEMIS-Th. Doc. Ing. Ecole Centrale (1983).

[5] Peri, G., Desautel, J., et al. Unités de conversion héliothermiques dans la gamme des moyennes puissances. Rev. COMPLES (1978) 27-31.

[6] PASQuetTi, R., La conversion héliothermique à moyenne température : sélectivité et concentration. Thèse d'Etat, Marseille (1983).

[7] Audibert, M., Programme solaire THEK. Production de chaleur à des fins industrielles, Rev. Gen. Th., 234-235 (1981) 509.

[8] Battistelli, J. P. et al., Optimisation des chaudières tubulaires planes ou en cavité. Application aux capteurs THEK, Rev. Gen. Th. 258-259 (1983) 453.

[9] Pasquetti, R., Suzzoni, J. et al., Etude en régime dynamique des convertisseurs héliothermiques tubulaires. Application aux capteurs THEK, Rev. Gen. Th. 270-271 (1984) 413.

[10] SoufiaNi, A., Absorption, émission et réflexion du rayonnement électromagnétique par une paroi constituée de tubes parallèles jointifs, Rev. Phys. Appl. 18 (1983) 19.
[11] Hottel, A. C., SARofim, A. F., Radiative transfer McGraw Hill (1967).

[12] DeVRIENDT, A. B., La transmission de la chaleur, Ed. Morin, 2 (1982).

[13] Pasquetti, R., Durastanti, J. F., et al. Modélisation numérique des sous-systèmes thermiques d'une centrale solaire de type THEK. Colloque Modélisation et Simulation en Thermique. Poitiers (1984).

[14] Reynolds, W. C., Turbulent heat transfer in a circular tube with variable circumferential heat flux, Int. J. Heat Mass Transfer, 6 (1963) 445.

[15] Sparrow, E. M., LiN S. H., Turbulent heat transfert in a tube with circumferentially-varying temperature or heat flux, Int. J. Heat Mass Transfer, 6 (1963) 866.

[16] Black, A. W., Sparrow, E. M., Experiments on turbulent heat transfer in a tube with circumferentially varying thermal boundary conditions, $J$. Heat Transfer (1967) 259.

[17] CADIOU, A., Répartition des températures dans un tuyau ou un ensemble de tuyaux axisymétriques contenant un fluide en écoulement et soumis à des conditions thermiques dissymétriques. Rev. Phys. Appl. 18 (1983) 153.

[18] GarTnER, D., et al. Turbulent heat transfer in a circular tube with circumferentially varying thermal boundary conditions, Int. J. Heat Mass Transfer 17 (1974).

[19] KAWAMURÁ, H., Experimental and analytical study of transient heat transfer for turbulent flow in a circular tube, Int. J. Heat Mass Transfer 20 (1977) 443.

[20] Knowles, G. R., Sparrow N. E. M., Local and average heat transfert characteristics for turbulent airflow in an asymmetrically heated tube, $J$. Heat Transfer 101 (1979) 635. 